This is the final peer-reviewed accepted manuscript of:

Detomi, E., Morigi, M., \& Shumyatsky, P. (2018). On profinite groups with word values covered by nilpotent subgroups. Israel Journal of Mathematics, 226(2).

The final published version is available online at:

http://dx.doi.org/10.1007/s11856-018-1720-2

Rights / License:

The terms and conditions for the reuse of this version of the manuscript are specified in the publishing policy. For all terms of use and more information see the publisher's website.

This item was downloaded from IRIS Università di Bologna (https://cris.unibo.it/)

When citing, please refer to the published version. 


\title{
On profinite groups with word values covered by nilpotent subgroups
}

\author{
Eloisa Detomi, Marta Morigi, and Pavel Shumyatsky
}

\begin{abstract}
Let $\mathcal{N}$ stand for the class of nilpotent groups or one of its wellknown generalizations. For a multilinear commutator word $w$ and a profinite group $G$ we show that $w(G)$ is finite-by- $\mathcal{N}$ if and only if the set of $w$-values in $G$ is covered by countably many finite-by- $\mathcal{N}$ subgroups. Earlier this was known only in the case where $w=x$ or $w=[x, y]$.
\end{abstract}

\section{Introduction}

In recent years profinite groups in which the set of word-values is covered by countably many subgroups with special properties attracted some interest (cf. [1]). Here we say that a set is covered by subgroups if it is contained in the set theoretical union of the subgroups. Given a group-word $w$ in $n$ variables and a group $G$, the verbal subgroup $w(G)$ of $G$ determined by the word $w$ is the subgroup generated by the set consisting of all values $w\left(g_{1}, \ldots, g_{n}\right)$, where $g_{1}, \ldots, g_{n}$ are elements of $G$. In the present paper we deal with the so called multilinear commutators (otherwise known under the name of outer commutator words). These are words which are obtained by nesting commutators, but using always different variables. For example, the word $\left[\left[x_{1}, x_{2}\right],\left[x_{3}, x_{4}, x_{5}\right], x_{6}\right]$ is a multilinear commutator while the Engel word $\left[x_{1}, x_{2}, x_{2}, x_{2}\right]$ is not.

A profinite group is a topological group that is isomorphic to an inverse limit of finite groups. In the context of profinite groups all the usual concepts of group theory are interpreted topologically. In particular, in a profinite group the verbal subgroup corresponding to the word $w$ is the closed subgroup generated by all $w$ values. More generally, in this paper by a subgroup of a profinite group we always mean a closed subgroup and by a quotient we mean a quotient over a normal closed subgroup.

In the present article we work with certain generalizations of nilpotent groups. Recall that a group $G$ is locally nilpotent if all finitely generated subgroups of $G$ are nilpotent. Following Shalev $[\mathbf{9}]$, we say that a group $G$ is strongly locally nilpotent if it belongs to a locally nilpotent variety of groups. This means that, for some function $f$ and for all positive integers $d$, every $d$-generated subgroup of $G$ is nilpotent of class at most $f(d)$. According to Wilson and Zelmanov a profinite group is locally nilpotent if and only if it is Engel [13]. Such a group is strongly locally nilpotent if it is $n$-Engel for some positive $n$ (see [12] or [14]).

Throughout the present article $\mathcal{N}$ stands for one of the following classes of groups.

2000 Mathematics Subject Classification. Primary 20E18; Secondary 20F12; $20 \mathrm{~F} 14$.

Key words and phrases. Profinite groups; Coverings; Commutators. 
- The class of nilpotent groups;

- The class of pronilpotent groups;

- The class of locally nilpotent groups;

- The class of strongly locally nilpotent groups.

The class of groups $G$ having a finite normal subgroup $D$ such that the quotient $G / D$ belongs to $\mathcal{N}$ is denoted by $\mathcal{F} \mathcal{N}$.

The main result of the present article can be stated as follows.

THEOREM 1.1. Let $w$ be a multilinear commutator word and let $G$ be a profinite group. The verbal subgroup $w(G)$ belongs to the class $\mathcal{F N}$ if and only if the set of $w$-values in $G$ is covered by countably many $\mathcal{F} \mathcal{N}$-subgroups.

This generalizes the results of $[\mathbf{1 1}]$ and [5] where similar conclusions were derived in the case where $w$ is either the word $x$ or the word $[x, y]$. Since a profinite group $G$ is in the class $\mathcal{F N}$ if and only if $G$ is covered by finitely many $\mathcal{N}$-subgroups (see $[\mathbf{1 1}, \mathbf{5}]$ ), we obtain the following corollary.

COROLlaRY 1.2. Let $w$ be a multilinear commutator word and let $G$ be a profinite group. The following statements are equivalent.

1. The verbal subgroup $w(G)$ belongs to the class $\mathcal{F} \mathcal{N}$;

2. The set of $w$-values in $G$ is covered by countably many $\mathcal{N}$-subgroups;

3. The set of $w$-values in $G$ is covered by finitely many $\mathcal{N}$-subgroups.

Theorem 1.1 and Corollary 1.2 are in parallel with results obtained earlier in $[3,4]$ that say that $w(G)$ is locally finite, or has finite rank, if and only if the set of $w$-values in $G$ is contained in a union of countably many subgroups with the respective property. Moreover, $w(G)$ is finite if and only if the set of $w$-values in $G$ is countable. In fact, the combinatorial techniques for handling multilinear commutator words developed in $[3,4]$ play an important role in the proof of Theorem 1.1. Unsurprisingly, the proof of Theorem 1.1 is much more complicated than the proofs in the case when $w=[x, y]$ in $[\mathbf{1 1}]$ and $[\mathbf{5}]$.

\section{Preliminary results}

In any group a product of finitely many normal $\mathcal{N}$-subgroups is again a normal $\mathcal{N}$-subgroup. This is well-known when $\mathcal{N}$ is the class of nilpotent, pronilpotent or locally nilpotent groups, while for the class of strongly locally nilpotent groups it is Lemma 2.4 in [5]. The following lemma extends this observation to products of normal $\mathcal{F} \mathcal{N}$-subgroups.

LEMMA 2.1. [5, Lemma 2.5] In any group a product of finitely many normal $\mathcal{F} \mathcal{N}$-subgroups is again in $\mathcal{F} \mathcal{N}$.

If $\mathcal{C}$ is a class of groups, a virtually- $\mathcal{C}$ group is a group with a normal $\mathcal{C}$-subgroup of finite index.

LEMMA 2.2. In any group a product of finitely many normal virtually- $\mathcal{F} \mathcal{N}$ subgroups is again a virtually- $\mathcal{F} \mathcal{N}$ subgroup.

Proof. It is sufficient to prove the lemma for a product of two normal virtually$\mathcal{F} \mathcal{N}$ subgroups $N_{1}$ and $N_{2}$ of a group $G$. By Lemma 2.1 , since $N_{i}$ is virtually- $\mathcal{F} \mathcal{N}$, there exists a unique maximal normal $\mathcal{F} \mathcal{N}$-subgroup $R_{i}$ of $N_{i}$ for each $i=1,2$. Then $R_{1}, R_{2}$ are normal in $G$ and so $R_{1} R_{2}$ is a normal $\mathcal{F} \mathcal{N}$-subgroup of finite index in $N_{1} N_{2}$.

If $A$ is a subset of a group $G$, we write $\langle A\rangle$ for the subgroup generated by $A$. If $B$ is another subset, we denote by $A^{B}$ the set $\left\{a^{b} \mid a \in A\right.$ and $\left.b \in B\right\}$. 
LEMma 2.3. [5, Lemma 2.6] Let $L$ be a subgroup of a profinite group $G$ such that the normalizer $N_{G}(L)$ is open.

(1) If $L$ is finite, then $\left\langle L^{G}\right\rangle$ is finite.

(2) If $L$ is in $\mathcal{F} \mathcal{N}$ and $H$ is a normal open subgroup of $G$ contained in $N_{G}(L)$, then $\left\langle(L \cap H)^{G}\right\rangle$ is in $\mathcal{F N}$.

The next two lemmas generalize [2, Lemma 2.1] and [2, Lemma 2.2] to the case of multilinear commutator words. Recall that the weight of a multilinear commutator $w$ is just the number of different variables involved in $w$.

LEMma 2.4. Let $w$ be a multilinear commutator word of weight $n$. Assume that $H$ is a normal subgroup of a group $G$. Let $g_{1}, \ldots, g_{n} \in G, h \in H$ and fix $s \in\{1, \ldots, n\}$. Then for every $j=1, \ldots, n$ there exist $y_{j} \in g_{j}^{H}$ such that

$$
\begin{array}{r}
w\left(g_{1}, \ldots, g_{s-1}, g_{s} h, g_{s+1}, \ldots, g_{n}\right)= \\
w\left(y_{1}, \ldots, y_{n}\right) w\left(g_{1}, \ldots, g_{s-1}, h, g_{s+1}, \ldots, g_{n}\right) .
\end{array}
$$

Proof. We argue by induction on $n$. If $n=1$, then the result is self-evident.

Assume that $n \geq 2$. Then there exist two multilinear commutator words $w_{1}$ and $w_{2}$ such that $w=\left[w_{1}, w_{2}\right]$. Let $l$ be the weight of $w_{1}$ and assume that $s \leq l$. By induction, for every $j \leq l$ there exist $y_{j} \in g_{j}^{H}$ such that

$$
\begin{gathered}
w_{1}\left(g_{1}, \ldots, g_{s-1}, g_{s} h, g_{s+1}, \ldots, g_{l}\right)= \\
w_{1}\left(y_{1}, \ldots, y_{l}\right) w_{1}\left(g_{1}, \ldots, g_{s-1}, h, g_{s+1}, \ldots, g_{l}\right) .
\end{gathered}
$$

Note that $\bar{h}=w_{1}\left(g_{1}, \ldots, g_{s-1}, h, g_{s+1}, \ldots, g_{l}\right) \in H$.

Using the standard commutator identities we compute

$$
\begin{gathered}
w\left(g_{1}, \ldots, g_{s-1}, g_{s} h, g_{s+1}, \ldots, g_{n}\right)=\left[w_{1}\left(y_{1}, \ldots, y_{l}\right) \bar{h}, w_{2}\left(g_{l+1}, \ldots, g_{n}\right)\right]= \\
{\left[w_{1}\left(y_{1}, \ldots, y_{l}\right), w_{2}\left(g_{l+1}, \ldots, g_{n}\right)\right]^{\bar{h}}\left[\bar{h}, w_{2}\left(g_{l+1}, \ldots, g_{n}\right)\right]=} \\
{\left[w_{1}\left(y_{1}^{\bar{h}}, \ldots, y_{l}\right), w_{2}\left(g_{l+1}^{\bar{h}}, \ldots, g_{n}^{\bar{h}}\right)\right]\left[\bar{h}, w_{2}\left(g_{l+1}, \ldots, g_{n}\right)\right],}
\end{gathered}
$$

and we obtain the desired result. The case $s>l$ is similar.

Let $w=w\left(x_{1}, \ldots, x_{n}\right)$ be a multilinear commutator word. If $A_{1}, \ldots, A_{n}$ are subsets of a group $G$, we write $w\left(A_{1}, \ldots, A_{n}\right)$ to denote the subgroup generated by the set of all $w$-values $w\left(a_{1}, \ldots, a_{n}\right)$ with $a_{i} \in A_{i}$.

Let $I$ be a subset of $\{1, \ldots, n\}$. Suppose that we have a family $A_{i_{1}}, \ldots, A_{i_{s}}$ of subsets of $G$ with indices running over $I$ and another family $B_{l_{1}}, \ldots, B_{l_{t}}$ of subsets with indices running over $\{1, \ldots, n\} \backslash I$. We write

$$
w_{I}\left(A_{i} ; B_{l}\right)
$$

for $w\left(X_{1}, \ldots, X_{n}\right)$, where $X_{k}=A_{k}$ if $k \in I$, and $X_{k}=B_{k}$ otherwise.

LEMma 2.5. Let $G$ be a group and let $w$ be a multilinear commutator of weight n. Assume that $H, A_{1}, \ldots, A_{n}$ are normal subgroups of $G$ such that for some elements $a_{i} \in A_{i}$, the equality

$$
w\left(a_{1}\left(H \cap A_{1}\right), \ldots, a_{n}\left(H \cap A_{n}\right)\right)=1
$$

holds. Then for any subset $I$ of $\{1, \ldots, n\}$ we have

$$
w_{I}\left(H \cap A_{i} ; a_{l}\left(H \cap A_{l}\right)\right)=1 .
$$

Proof. The proof is by induction on the size of $I$, the case $I=\emptyset$ being trivial. Let $I$ be a non-empty subset of $\{1, \ldots, n\}$ and fix $s \in I$. By induction applied to $I^{*}=I \backslash\{s\}$ we have

$$
w_{I^{*}}\left(H \cap A_{i} ; a_{l}\left(H \cap A_{l}\right)\right)=1 .
$$


Let $\bar{w}=w\left(g_{1}, \ldots, g_{n}\right)$, where $g_{i} \in H \cap A_{i}$ if $i \in I$ and $g_{i} \in a_{i}\left(H \cap A_{i}\right)$ otherwise. It suffices to prove that $\bar{w}=1$.

We apply Lemma 2.4 to the element obtained by replacing in $\bar{w}$ the entry $g_{s}$ with $a_{s} g_{s}$ : since $g_{s} \in H$, there exist elements $y_{i} \in g_{i}^{H} \subseteq g_{i}\left(H \cap A_{i}\right)$ for every $i \neq s$ and $y_{s} \in a_{s}^{H} \subseteq a_{s}\left(H \cap A_{s}\right)$ such that

$$
\begin{aligned}
w\left(g_{1}, \ldots, g_{s-1}, a_{s} g_{s}, g_{s+1}, \ldots, g_{n}\right) & =w\left(y_{1}, \ldots, y_{n}\right) w\left(g_{1}, \ldots, g_{n}\right) \\
& =w\left(y_{1}, \ldots, y_{n}\right) \bar{w} .
\end{aligned}
$$

Thus, $g_{i}, y_{i} \in H \cap A_{i}$ when $i \in I^{*}$ and $g_{l}, y_{l} \in a_{l}\left(H \cap A_{l}\right)$ when $l \notin I$. Since $y_{s} \in a_{s}\left(H \cap A_{s}\right)$, by assumption (1) we have

$$
w\left(g_{1}, \ldots, g_{s-1}, a_{s} g_{s}, g_{s+1}, \ldots, g_{n}\right) \in w_{I^{*}}\left(H \cap A_{i} ; a_{l}\left(H \cap A_{l}\right)\right)=1,
$$

and

Therefore $\bar{w}=1$.

Corollary 2.6. Let $G$ be a group and let $w$ be a multilinear commutator of weight $n$. Assume that $H$ is a normal subgroup of $G$ such that for some elements $a_{1}, \ldots, a_{n} \in G$ the equality $w\left(a_{1} H, \ldots, a_{n} H\right)=1$ holds. Then $w(H)=1$.

We recall that an element of a group $G$ is called an $F C$-element if it has only finitely many conjugates in $G$.

LEMMA 2.7. Let $G=\left\langle H, a_{1}, \ldots, a_{s}\right\rangle$ be a group, where $H$ is a finite index abelian normal subgroup and $a_{1}, \ldots, a_{s}$ are $F C$-elements. Then $G^{\prime}$ is finite.

Proof. Note that $H \cap C_{G}\left(a_{1}\right) \cap \cdots \cap C_{G}\left(a_{s}\right)$ is contained in the center of $G$ and has finite index in $G$. So the result follows from Schur's theorem $[8,10.1 .4]$.

\section{The case of derived words}

An important family of multilinear commutator words is formed by so-called derived words $\delta_{k}$, on $2^{k}$ variables, defined recursively by

$$
\delta_{0}=x_{1}, \quad \delta_{k}=\left[\delta_{k-1}\left(x_{1}, \ldots, x_{2^{k-1}}\right), \delta_{k-1}\left(x_{2^{k-1}+1}, \ldots, x_{2^{k}}\right)\right] .
$$

Of course $\delta_{k}(G)=G^{(k)}$ is the $k$-th term of the derived series of $G$.

In the present section we deal with groups in which $\delta_{k}$-values are covered by countably many $\mathcal{F} \mathcal{N}$-subgroups. We also develop techniques that will be helpful in handling the general case of Theorem 1.1 in the subsequent section.

Lemma 3.1. [5, Lemma 3.2] Let $G=N\langle b\rangle$ be a profinite group where $N$ is an open normal $\mathcal{F} \mathcal{N}$-subgroup of $G$. Assume that there exists an open normal subgroup $R$ of $G$ such that $R \leq N$ and $R\langle b\rangle$ is in $\mathcal{F N}$. Then $G$ is in $\mathcal{F N}$.

In the sequel we will often use without mentioning the fact that if $w$ is a word, the conjugate of a $w$-value is again a $w$-value. Next lemma holds for any word $w$.

LEMmA 3.2. Let $w$ be a word and let $G$ be a profinite group in which the set of $w$-values is covered by countably many $\mathcal{F} \mathcal{N}$-subgroups $G_{i}$. Suppose that $x$ is a $w$-value and $N$ is a normal $\mathcal{F} \mathcal{N}$-subgroup of $G$ such that $N$ is open in $\langle N, x\rangle$. Then the subgroup $\langle N, x\rangle$ is in $\mathcal{F N}$.

Proof. Let $X$ be the set of all $w$-values contained in the coset $x N$. Of course $X$ is non-empty. Obviously, the set $X$ is closed and therefore compact. It is clear that $X$ is covered by the (closed) subsets $X \cap G_{i}$. By the Baire category theorem (cf [7, p. 200]), at least one of these subsets has non-empty interior. Hence, there exist an open normal subgroup $T$ of $G$, an element $b \in X$ and an index $j$ such that $X \cap b T$ is a subset of $G_{j}$. Let $R=T \cap N$. Notice that for every $r \in R$, the 
conjugate $b^{r}$ is a $w$-value and $b^{r}=b[b, r] \in b R$. Since all $w$-values contained in $b R$ belong to $G_{j}$, it follows that $\left\langle b^{R}\right\rangle \leq G_{j}$. So $\left\langle b^{R}\right\rangle$ is in $\mathcal{F N}$. We observe that $\langle b, R\rangle=\left\langle b^{R}\right\rangle R$ is a product of two normal $\mathcal{F} \mathcal{N}$-subgroups so it is in $\mathcal{F} \mathcal{N}$ by Lemma 2.1. Since $R$ is open in $N$, in view of Lemma 3.1 we conclude that $\langle N, b\rangle$ is in $\mathcal{F N}$. As $\langle N, b\rangle=\langle N, x\rangle$, the lemma follows.

Throughout the rest of the article we will work under the following hypothesis.

Hypothesis 3.3. Let $w$ be a multilinear commutator of weight $n$ and let $G$ be a profinite group in which the set of $w$-values of $G$ is contained in a union of countably many $\mathcal{F} \mathcal{N}$-subgroups $G_{i}$.

LEMma 3.4. Assume Hypothesis 3.3. Then G contains an open normal subgroup $H$ such that $w(H)$ is virtually- $\mathcal{F} \mathcal{N}$.

Proof. For each positive integer $i$ consider the set

$$
S_{i}=\left\{\left(g_{1}, \ldots, g_{n}\right) \in G \times \cdots \times G \mid w\left(g_{1}, \ldots, g_{n}\right) \in G_{i}\right\} .
$$

Note that the sets $S_{i}$ are closed in $G \times \cdots \times G$ and cover the whole group $G \times \cdots \times G$. By the Baire category theorem at least one of these sets has non-empty interior. Hence, there exist an open normal subgroup $H$ of $G$, elements $a_{1}, \ldots, a_{n} \in G$, and an integer $j$ such that $w\left(a_{1} H, \ldots, a_{n} H\right) \subseteq G_{j}$.

Let

$$
K=w\left(a_{1} H, \ldots, a_{n} H\right) .
$$

Note that $K \leq G_{j}$ and $H$ normalizes $K$. Since $G_{j}$ is in $\mathcal{F} \mathcal{N}$, so is $K$. Let $D=K \cap H$. By Lemma 2.3, $\left\langle D^{G}\right\rangle$ is in $\mathcal{F N}$.

Note that $\left\langle D^{G}\right\rangle$ has finite index in $\left\langle K^{G}\right\rangle$. Indeed, suppose that $D=1$. In this case $K$ is a finite subgroup normalized by $H$ and thus $\left\langle K^{G}\right\rangle$ is finite. Hence $\left\langle D^{G}\right\rangle$ has finite index in $\left\langle K^{G}\right\rangle$. It follows that $\left\langle K^{G}\right\rangle$ is virtually- $\mathcal{F} \mathcal{N}$. The quotient $\bar{G}=G /\left\langle K^{G}\right\rangle$ satisfies the hypothesis of Corollary 2.6, whence $w(\bar{H})=1$. It follows that $w(H) \leq\left\langle K^{G}\right\rangle$ and hence it is virtually- $\mathcal{F} \mathcal{N}$.

Lemma 3.5. Assume Hypothesis 3.3 and let $a \in G$ be $a w$-value. Then there exists a normal open subgroup $H_{a}$ of $G$ such that $\left[H_{a}, a\right]$ is in $\mathcal{F N}$.

Proof. For each positive integer $i$ let

$$
S_{i}=\left\{x \in G \mid a^{x} \in G_{i}\right\} .
$$

Note that the sets $S_{i}$ are closed in $G$ and cover the whole group $G$. By the Baire category theorem at least one of these sets has non-empty interior. Hence, there exist an open normal subgroup $H$ of $G$, an element $b \in G$, and an integer $j$ such that $b H \leq S_{j}$, i.e. $a^{b h} \in G_{j}$ for any $h \in H$. Thus $\left\langle a^{b H}\right\rangle \leq G_{j}$. Since $\left[a^{b}, H\right] \leq\left\langle a^{b H}\right\rangle$, we conclude that $\left[a, H^{b^{-1}}\right] \leq G_{j}^{b^{-1}}$, and the result follows. $\mathcal{F N}$.

Proposition 3.6. Assume Hypothesis 3.3 with $w=\delta_{i}$. Then $G^{(2 i)}$ is virtually-

Proof. By Lemma 3.4 there exists an open normal subgroup $H$ such that $H^{(i)}$ is virtually- $\mathcal{F} \mathcal{N}$. Let $K=G^{(i)}, L=K \cap H$. Note that $L$ is open in $K$. Choose a finite set of $\delta_{i}$-values $a_{1}, \ldots, a_{s}$ such that $K=\left\langle L, a_{1}, \ldots, a_{s}\right\rangle$ and let $H_{a_{1}}, \ldots, H_{a_{s}}$ be normal open subgroups of $G$ such that $\left[H_{a_{j}}, a_{j}\right]$ is in $\mathcal{F} \mathcal{N}$ for every $j$ (see Lemma 3.5). Note that for each $j$ the subgroup $\left[H_{a_{j}}, a_{j}\right]$ is a normal subgroup of $H_{a_{j}}$ so, by Lemma 2.3, $\left\langle\left[H_{a_{j}}, a_{j}\right]^{G}\right\rangle$ is in $\mathcal{F N}$. Let $N_{1} \leq G^{(i)}$ be the subgroup generated by $L^{(i)}$ and the subgroups $\left\langle\left[H_{a_{j}}, a_{j}\right]^{G}\right\rangle$ for $j=1, \ldots, s$. Note that $N_{1}$ is virtually- $\mathcal{F N}$ by Lemma 2.2. The images of $a_{1}, \ldots, a_{s}$ in the quotient $G / N_{1}$ are $F C$-elements while the image of $L$ in $G / L^{\prime}$ is abelian. Therefore by Lemma 
2.7 the group $K N_{1} / L^{\prime} N_{1}$ has finite derived group. In other words $L^{\prime} N_{1}$ has finite index in $K^{\prime} N_{1}$. In particular there exist finitely many $\delta_{i}$-values $b_{1}, \ldots, b_{t}$ such that $K^{\prime} N_{1}=\left\langle L^{\prime}, b_{1}, \ldots, b_{t}, N_{1}\right\rangle$.

As above, there exist normal open subgroups $H_{b_{1}}, \ldots, H_{b_{t}}$ of $G$ such that $\left\langle\left[H_{b_{j}}, b_{j}\right]^{G}\right\rangle$ is in $\mathcal{F} \mathcal{N}$ for every $j$. Let $N_{2}$ be the subgroup generated by $N_{1}$ and the subgroups $\left\langle\left[H_{b_{j}}, b_{j}\right]^{G}\right\rangle$ for $j=1, \ldots, t$. Note that $N_{2}$ is virtually- $\mathcal{F} \mathcal{N}$ by Lemma 2.2. Again, $b_{1} N_{2}, \ldots, b_{t} N_{2}$ are FC-elements in $G / N_{2}$ and arguing as before we obtain that $L^{(2)} N_{2}$ has finite index in $K^{(2)} N_{2}$. By iterating this argument we get that $L^{(i)} N_{i}$ has finite index in $K^{(i)} N_{i}$ for some normal virtually- $\mathcal{F} \mathcal{N}$ subgroup $N_{i}$, so $L^{(i)}\left(K^{(i)} \cap N_{i}\right)$ has finite index in $K^{(i)}=G^{(2 i)}$. As $L^{(i)} \leq H^{(i)}$ is virtually- $\mathcal{F} \mathcal{N}$ it follows that $G^{(2 i)}$ is virtually- $\mathcal{F N}$, as desired.

Proposition 3.7. Assume Hypothesis 3.3 with $w=\delta_{k}$. Suppose that $G^{(k)}$ is virtually- $\mathcal{F} \mathcal{N}$. Then $G^{(k)}$ is in $\mathcal{F N}$.

Proof. Let $N$ be an open characteristic $\mathcal{F} \mathcal{N}$-subgroup of $G^{(k)}$ and let $X$ be the set of $\delta_{k}$-values in $G$. Lemma 3.2 tells us that for each $x \in X$ the subgroup $\langle N, x\rangle$ is in $\mathcal{F} \mathcal{N}$. Let $D_{x}$ be the (unique) minimal characteristic finite subgroup of $\langle N, x\rangle$ such that $\langle N, x\rangle / D_{x}$ is in $\mathcal{N}$. Since $N$ is open in $G^{(k)}$, it follows that there are only finitely many subgroups of the form $\langle N, x\rangle$, where $x \in X$. Therefore $D_{x}$ has only finitely many conjugates in $G$ and so the normal closure of $D_{x}$ in $G$ is finite. It follows that $D=\left\langle D_{x}, x \in X\right\rangle$ is a finite normal subgroup of $G$. So we pass to the quotient $G / D$ and we can assume that $\langle N, x\rangle$ is in $\mathcal{N}$ for every $x \in X$.

Let $\pi$ be the set of primes dividing the order of $G^{(k)} / N$.

Suppose first that $N$ is a pro- $\pi^{\prime}$ group. Let $S$ be the set of all $\pi$-elements contained in procyclic subgroups generated by elements from $X$. Then $G^{(k)}=$ $N\langle S\rangle$. As $\langle N, x\rangle$ is in $\mathcal{N}$ for every $x \in X$, it follows that $N$ centralizes $\langle S\rangle$. Therefore the center of $\langle S\rangle$ has finite index in $\langle S\rangle$. So by Schur's theorem the derived group $\langle S\rangle^{\prime}$ is finite. As $\langle S\rangle$ is characteristic in $G$, we can pass to the quotient $G /\langle S\rangle^{\prime}$ and we may assume that $\langle S\rangle$ is abelian. Now $G^{(k)}$ is the product of two normal $\mathcal{N}$-subgroups, so it is in $\mathcal{N}$. This concludes the proof in the case when $N$ is a pro- $\pi^{\prime}$ group.

Now suppose that $N$ is a pro- $p$ group for some prime $p \in \pi$. Let $S_{1}$ be the set of all $p^{\prime}$-elements contained in procyclic subgroups generated by elements from $X$. Again, $N$ centralizes $S_{1}$, so $\left\langle S_{1}\right\rangle^{\prime}$ is finite. As above, we can assume that $\left\langle S_{1}\right\rangle$ is abelian. Therefore $\left\langle S_{1}\right\rangle$ is a $p^{\prime}$-subgroup of $G^{(k)}$. Since $G^{(k)}$ is virtually pro- $p$, it follows that $\left\langle S_{1}\right\rangle$ is finite, so by passing to the quotient $G /\left\langle S_{1}\right\rangle$ we can assume that all $\delta_{k}$-values of $G$ are $p$-elements. Using a profinite version of Lemma 3.1 in [10] we obtain that $G^{(k)}$ is a pro- $p$ group. Now we will prove that $G^{(k)}$ is in $\mathcal{N}$ by induction on $\left|G^{(k)}: N\right|$. Since $G^{(k)} / N$ is nilpotent, there is an index $i$ such that $\gamma_{i}\left(G^{(k)}\right) N / N$ is a nontrivial subgroup of the center of $G^{(k)} / N$ (here, as usual, $\gamma_{i}\left(G^{(k)}\right)$ denotes the $i$-th term of the lower central series of $\left.G^{(k)}\right)$. Notice that if $x_{1}, \ldots, x_{i} \in X$, then $\left[x_{1}, \ldots, x_{i}\right] \in X$. As $\gamma_{i}\left(G^{(k)}\right)$ is generated by $\gamma_{i}$-values whose entries are $\delta_{k}$-values, there exists a $\delta_{k}$-value $x$ such that $x N$ is a nontrivial element of the center of $G^{(k)} / N$. Therefore $\langle N, x\rangle$ is a normal $\mathcal{N}$-subgroup of $G^{(k)} / N$. The characteristic closure $M$ of $\langle N, x\rangle$ in $G$ is obviously again in $\mathcal{F N}$ and $\left|G^{(k)}: M\right|$ is smaller than $\left|G^{(k)}: N\right|$. Then, by induction we conclude that $G^{(k)}$ is in $\mathcal{F N}$.

Let $\pi=\left\{p_{1}, \ldots, p_{s}\right\}$ and let $N_{\pi}$ and $N_{p^{\prime}}$ be the Hall $\pi$-subgroup and the Hall $p^{\prime}$-subgroup of $N$, respectively. Since $N$ is pronilpotent, the subgroups $N_{\pi}, N_{p_{i}^{\prime}}$ are normal in $G$. We already know that all quotients $G^{(k)} / N_{\pi}$ and $G^{(k)} / N_{p_{i}^{\prime}}$ are in $\mathcal{F N}$. Moreover, $G^{(k)}$ is isomorphic to a subgroup of the direct product

$$
G^{(k)} / N_{\pi} \times G^{(k)} / N_{p_{1}^{\prime}} \times \cdots \times G^{(k)} / N_{p_{s}^{\prime}},
$$


which is the product of finitely many $\mathcal{F} \mathcal{N}$-groups. We conclude that $G^{(k)}$ is in $\mathcal{F N}$, as desired.

Corollary 3.8. Assume Hypothesis 3.3 with $w=\delta_{i}$. Then $G^{(2 i)}$ is in $\mathcal{F} \mathcal{N}$.

Proof. By Proposition 3.6 the subgroup $G^{(2 i)}$ is virtually- $\mathcal{F N}$. Then note that every $\delta_{2 i}$-value is in particular a $\delta_{i}$-value, so the hypotheses of Proposition 3.7 are satisfied when $k=2 i$ and we obtain the desired result.

\section{The general case}

In the present section we complete the proof of Theorem 1.1.

Recall the notation introduced in Section 2: whenever $I$ is a subset of $\{1, \ldots, n\}$ and $A_{i_{1}}, \ldots, A_{i_{s}}$ and $B_{l_{1}}, \ldots, B_{l_{t}}$ are families of subsets of $G$ with indices running over $I$ and $\{1, \ldots, n\} \backslash I$, respectively, we write

$$
w_{I}\left(A_{i} ; B_{l}\right)
$$

for the subgroup $w\left(X_{1}, \ldots, X_{n}\right)$, where $X_{k}=A_{k}$ if $k \in I$, and $X_{k}=B_{k}$ otherwise. On the other hand, whenever $a_{i} \in A_{i}$ for $i \in I$ and $b_{l} \in B_{l}$ for $l \in\{1, \ldots, n\} \backslash I$, the symbol $w_{I}\left(a_{i} ; b_{l}\right)$ stands for the element $w\left(x_{1}, \ldots, x_{n}\right)$, where $x_{k}=a_{k}$ if $k \in I$, and $x_{k}=b_{k}$ otherwise.

Lemma 4.1. Let $A_{1}, \ldots, A_{n}$ and $H$ be normal subgroups of a group $G$. Let I be a subset of $\{1, \ldots, n\}$. Assume that for every proper subset $J$ of $I$

$$
w_{J}\left(A_{i} ; H \cap A_{l}\right)=1 .
$$

Suppose we are given elements $g_{i} \in A_{i}$ with $i \in I$ and elements $h_{k} \in H \cap A_{k}$ with $k \in\{1, \ldots, n\}$. Then we have

$$
w_{I}\left(g_{i} h_{i} ; h_{l}\right)=w_{I}\left(g_{i} ; h_{l}\right) .
$$

Proof. Let

$$
\bar{w}=w_{I}\left(g_{i} h_{i} ; h_{l}\right)=w\left(c_{1}, \ldots, c_{n}\right)
$$

where $c_{i}=g_{i} h_{i}$ if $i \in I$, and $c_{i}=h_{i}$ otherwise.

Fix an index $s \in I$ and let $J=I \backslash\{s\}$. We can write $g_{s} h_{s}=\bar{h} g_{s}$ where $\bar{h}=h_{s}^{g_{s}^{-1}} \in H \cap A_{s}$. Then, by Lemma 2.4,

$$
\begin{aligned}
\bar{w} & =w\left(c_{1}, \ldots, c_{s-1}, \bar{h} g_{s}, c_{s+1}, \ldots, c_{n}\right) \\
& =w\left(y_{1}, \ldots, y_{n}\right) w\left(c_{1}, \ldots, c_{s-1}, g_{s}, c_{s+1}, \ldots, c_{n}\right)
\end{aligned}
$$

where $y_{s} \in \bar{h}^{G} \leq H \cap A_{s}$ and $y_{k} \in c_{k}^{G}$ for every $k \neq s$. In particular $y_{k} \in A_{k}$ if $k \in J$ and $y_{k} \in H \cap A_{k}$ if $k \notin I$. Therefore

$$
w\left(y_{1}, \ldots, y_{n}\right) \in w_{J}\left(A_{i} ; H \cap A_{l}\right)
$$

and so $w\left(y_{1}, \ldots, y_{n}\right)=1$ by assumption. Hence

$$
\bar{w}=w\left(c_{1}, \ldots, c_{s-1}, g_{s}, c_{s+1}, \ldots, c_{n}\right) .
$$

By repeating the argument for every $s \in I$, we get the desired conclusion.

Lemma 4.2. Assume Hypothesis 3.3. Let $T$ be a normal $\mathcal{F} \mathcal{N}$-subgroup of $G$ and let $A_{1}, \ldots, A_{n}$ be normal subgroups of $G$ such that $w\left(A_{1}, \ldots, A_{n}\right) T / T$ is abelian. Let $I$ be a subset of $\{1, \ldots, n\}$ and assume that $G$ has an open normal subgroup $H$ such that

(**) $w_{J}\left(A_{i} ; H \cap A_{l}\right) \leq T$, for every proper subset $J$ of $I$.

Then, for any given set of elements $\left\{g_{i}\right\}_{i \in I}$, where $g_{i} \in A_{i}$, there exist an open normal subgroup $U$ of $G$, contained in $H$, and a normal $\mathcal{F} \mathcal{N}$-subgroup $N$ of $G$, containing $T$, such that

$$
w_{I}\left(g_{i} ; U \cap A_{l}\right) \leq N
$$


Proof. Consider the sets

$$
S_{j}=\left\{\left(h_{1}, \ldots, h_{n}\right) \mid h_{k} \in H \cap A_{k} \text { and } w_{I}\left(g_{i} h_{i} ; h_{l}\right) \in G_{j}\right\} .
$$

Note that the sets $S_{j}$ are closed in the group $\left(H \cap A_{1}\right) \times \cdots \times\left(H \cap A_{n}\right)$ and cover the whole group. By the Baire category theorem at least one of these sets has non-empty interior. Hence, there exist an integer $r$, open subgroups $V_{k}$ of $H \cap A_{k}$, and elements $b_{k} \in H \cap A_{k}$ for every $k=1, \ldots, n$ such that

$$
w_{I}\left(g_{i} b_{i} V_{i} ; b_{l} V_{l}\right) \subseteq G_{r} .
$$

Each subgroup $V_{k}$ is of the form $V_{k}=U_{k} \cap H \cap A_{k}$ where $U_{k}$ is an open subgroup of $G$ and we can assume that $U_{k}$ is normal in $G$. Let $U=U_{1} \cap \cdots \cap U_{n} \cap H$. Note that $U$ is an open normal subgroup of $G$ contained in $H$. Now let

$$
K=w_{I}\left(g_{i} b_{i}\left(U \cap A_{i}\right) ; b_{l}\left(U \cap A_{l}\right)\right) .
$$

Then $K \subseteq G_{r}$ is in $\mathcal{F N}$ and $U$ normalizes $K$. Let $D=K \cap U$. Since $U$ has finite index in $\bar{G}$, by Lemma $2.3,\left\langle D^{G}\right\rangle$ is in $\mathcal{F} \mathcal{N}$. So we can assume that $\left\langle D^{G}\right\rangle \leq T$.

Set $R=\left\langle K^{G}\right\rangle$. Let us examine the quotient $\tilde{G}=G /\left\langle D^{G}\right\rangle$. We see that $\tilde{K}=K\left\langle D^{G}\right\rangle /\left\langle D^{G}\right\rangle$ is a finite subgroup normalized by $\tilde{U}$. Thus $\tilde{R}$ is finite. Note that $T \cap R$ has finite index in $R$. Moreover, $R /(T \cap R)$ is isomorphic to $R T / T \leq$ $w\left(A_{1}, \ldots, A_{n}\right) T / T$ which is abelian. As $R$ is generated by $w$-values, it is the product of finitely many subgroups of the form $\langle T \cap R, x\rangle$, where $x$ is a $w$-value. The subgroups $\langle T \cap R, x\rangle$ normalize each other and each of them is in $\mathcal{F N}$ by Lemma 3.2. It follows from Lemma 2.1 that $R$ is in $\mathcal{F N}$.

Let $N=R T$. Now in the quotient group $G / N$ the equality

$$
w_{I}\left(g_{i} b_{i}\left(U \cap A_{i}\right) ; b_{l}\left(U \cap A_{l}\right)\right)=1
$$

holds. In view of Lemma 2.5 we deduce that

$$
w_{I}\left(g_{i} b_{i}\left(U \cap A_{i}\right) ; U \cap A_{l}\right)=1
$$

in $G / N$. By condition $\left({ }^{* *}\right)$, given that $T \leq N$, we can apply Lemma 4.1 and we obtain that

$$
w_{I}\left(g_{i} ; U \cap A_{l}\right)=w_{I}\left(g_{i} b_{i}\left(U \cap A_{i}\right) ; U \cap A_{l}\right)=1,
$$

in $G / N$, that is,

as desired.

$$
w_{I}\left(g_{i} ; U \cap A_{l}\right) \leq N
$$

Lemma 4.3. Assume Hypothesis 3.3. Let $T$ be a normal $\mathcal{F} \mathcal{N}$-subgroup of $G$ and let $A_{1}, \ldots, A_{n}$ be normal subgroups of $G$ such that $w\left(A_{1}, \ldots, A_{n}\right) T / T$ is abelian. Let $I$ be a subset of $\{1, \ldots, n\}$ and assume that $G$ has an open normal subgroup $H$ such that

(**) $\quad w_{J}\left(A_{i} ; H \cap A_{l}\right) \leq T$, for every proper subset $J$ of $I$.

Then there exist an open normal subgroup $U$ of $G$, contained in $H$, and a normal $\mathcal{F} \mathcal{N}$-subgroup $N$ of $G$, containing $T$, such that

$$
w_{I}\left(A_{i} ; U \cap A_{l}\right) \leq N .
$$

Proof. For each $i \in I$ choose a set $R_{i}$ of coset representatives of $H \cap A_{i}$ in $A_{i}$. Note that all those sets are finite. Now we apply Lemma 4.2 to each choice of elements $\bar{g}=\left\{g_{i}\right\}_{i \in I}$, with $g_{i} \in R_{i}$ : let $U_{\bar{g}}$ and $N_{\bar{g}}$ be the normal subgroups of $G$ such that $w_{I}\left(g_{i} ; U_{\bar{g}} \cap A_{l}\right) \leq N_{\bar{g}}$. Note that there is only a finite number of $U_{\bar{g}}$ 's and $N_{\bar{g}}$ 's. Then $U=\cap_{\bar{g}} U_{\bar{g}}$ is a normal open subgroup of $G$ contained in $H$ and $N=\prod_{\bar{g}} N_{\bar{g}}$ is a normal $\mathcal{F} \mathcal{N}$-subgroup containing $T$, such that

$$
w_{I}\left(g_{i} ; U \cap A_{l}\right) \leq N
$$


for every choice of $g_{i} \in R_{i}$. Note that, by condition (**) and Lemma 4.1,

$$
w_{I}\left(g_{i}\left(H \cap A_{i}\right) ; U \cap A_{l}\right)=w_{I}\left(g_{i} ; U \cap A_{l}\right) \leq N .
$$

Since $A_{i}=\cup_{g_{i} \in R_{i}} g_{i}\left(H \cap A_{i}\right)$ for every $i \in I$, we conclude that

$$
w_{I}\left(A_{i} ; U \cap A_{l}\right)=\left\langle\cup_{\bar{g}} w_{I}\left(g_{i}\left(H \cap A_{i}\right) ; U \cap A_{l}\right)\right\rangle \leq N,
$$

as desired.

Lemma 4.4. Assume Hypothesis 3.3. Suppose that there exists an open normal subgroup $H$ of $G$ such that $w(H) \leq T$, where $T$ is a normal $\mathcal{F} \mathcal{N}$-subgroup of $G$. Let $A_{1}, \ldots, A_{n}$ be normal subgroups of $G$ such that in the quotient group $G / T$ the subgroup $w\left(A_{1}, \ldots, A_{n}\right) T / T$ is abelian. Then $w\left(A_{1}, \ldots, A_{n}\right)$ is in $\mathcal{F} \mathcal{N}$.

Proof. It is enough to prove the following statement: for every subset $I$ of $\{1, \ldots, n\}$, there exist an open normal subgroup $U_{I}$ of $G$ contained in $H$ and a normal $\mathcal{F} \mathcal{N}$-subgroup $N_{I}$ containing $T$ such that $w_{I}\left(A_{i} ; U_{I} \cap A_{l}\right) \leq N_{I}$.

The proof is by induction on the size $k$ of $I$. If $k=0$, then $I=\emptyset$ and

$$
w_{\emptyset}\left(A_{i} ; H \cap A_{i}\right)=w\left(H \cap A_{1}, \ldots, H \cap A_{n}\right) \leq w(H) \leq T .
$$

So assume $k>0$. Let $J_{1}, \ldots, J_{s}$ be all proper subsets of $I$. By induction, for each $t=1, \ldots, s$ there exist an open normal subgroup $U_{t}$ of $G$ contained in $H$ and a normal $\mathcal{F} \mathcal{N}$-subgroup $N_{t}$ containing $T$ such that $w_{J_{t}}\left(A_{i} ; U_{t} \cap A_{l}\right) \leq N_{t}$.

Let $U=\cap_{t} U_{t}$ and $N=\left\langle N_{t}, t=1, \ldots, s\right\rangle$. Then $w_{J}\left(A_{i} ; U \cap A_{l}\right) \leq N$, for every proper subgroup $J$ of $I$. Now, we can apply Lemma 4.3 to $I$. We obtain that there exist an open normal subgroup $U_{I}$ of $G$ contained in $H$ and a normal $\mathcal{F} \mathcal{N}$-subgroup $N_{I}$ containing $T$ such that $w_{I}\left(A_{i} ; U_{I} \cap A_{l}\right) \leq N_{I}$, as desired.

We denote by $\mathbf{I}$ the set of all $n$-tuples $\left(i_{1}, \ldots, i_{n}\right)$, where all entries $i_{k}$ are nonnegative integers. We will view $\mathbf{I}$ as a partially ordered set with the partial order given by the rule that

$$
\left(i_{1}, \ldots, i_{n}\right) \leq\left(j_{1}, \ldots, j_{n}\right)
$$

if and only if $i_{1} \leq j_{1}, \ldots, i_{n} \leq j_{n}$.

Given $\mathbf{i}=\left(i_{1}, \ldots, i_{n}\right) \in \mathbf{I}$, we write

$$
w(\mathbf{i})=w\left(G^{\left(i_{1}\right)}, \ldots, G^{\left(i_{n}\right)}\right)
$$

for the subgroup generated by the $w$-values $w\left(g_{1}, \ldots, g_{n}\right)$ with $g_{j} \in G^{\left(i_{j}\right)}$. Further, let

$$
w\left(\mathbf{i}^{+}\right)=\prod w(\mathbf{j}),
$$

where the product is taken over all $\mathbf{j} \in \mathbf{I}$ such that $\mathbf{j}>\mathbf{i}$.

Lemma 4.5. [3, Corollary 6] Let $w=w\left(x_{1}, \ldots, x_{n}\right)$ be a multilinear commutator word and let $\mathbf{i} \in \mathbf{I}$. If $w\left(\mathbf{i}^{+}\right)=1$, then $w(\mathbf{i})$ is abelian.

We will need the following well-known result (see for example [10, Lemma 4.1]).

LEMmA 4.6. Let $G$ be a group and let $w$ be a multilinear commutator word on $n$ variables. Then each $\delta_{n}$-value is a $w$-value.

Lemma 4.7. Assume Hypothesis 3.3. If $w(G)$ is virtually- $\mathcal{F} \mathcal{N}$ then $w(G)$ is in $\mathcal{F N}$.

Proof. Assume that $w(G)$ is virtually- $\mathcal{F} \mathcal{N}$. It follows from Lemma 2.1 that there exists a maximal open normal $\mathcal{F} \mathcal{N}$-subgroup $R$ of $w(G)$. By Lemma 4.6 every $\delta_{n}$-value in $G$ is a $w$-value. In view of Corollary 3.8 we deduce that $G^{(2 n)}$ is in $\mathcal{F} \mathcal{N}$. Hence $G^{(2 n)} \leq R$. Since $G / R$ is soluble, there exist only finitely many $\mathbf{i} \in \mathbf{I}$ such that $w(\mathbf{i}) R / R \neq 1$. 
Assume, by contradiction, that $w(G) R \neq R$ and choose $\mathbf{i}=\left(i_{1}, \ldots, i_{n}\right) \in \mathbf{I}$ such that $w(\mathbf{i}) R / R \neq 1$ while $w(\mathbf{j}) R / R=1$ whenever $\mathbf{i}<\mathbf{j}$. By Lemma $4.5, w(\mathbf{i}) R / R$ is abelian. As $w(\mathbf{i})$ is generated by $w$-values, $w(\mathbf{i}) R$ is a product of finitely many normal subgroups of the form $\langle R, x\rangle$, where $x$ is a $w$-value. Each subgroup $\langle R, x\rangle$ is in $\mathcal{F N}$ by Lemma 3.2. It follows that $w(\mathbf{i}) R$ is in $\mathcal{F N}$. Therefore $w(\mathbf{i}) \leq R$, a contradiction.

We are now ready to complete the proof of Theorem 1.1.

\section{Proof of Theorem 1.1}

We only need to show that if $w=w\left(x_{1}, \ldots, x_{n}\right)$ is a multilinear commutator word and $G$ is a profinite group in which the set of $w$-values is covered by countably many $\mathcal{F} \mathcal{N}$-subgroups then $w(G)$ is in $\mathcal{F N}$. By Lemma 4.6 every $\delta_{n}$-value in $G$ is a $w$-value. In view of Corollary 3.8 we deduce that $G^{(2 n)}$ is in $\mathcal{F N}$.

Let $H$ be as in Lemma 3.4. By Lemma 4.7, $w(H)$ is in $\mathcal{F N}$. Let $T=$ $G^{(2 n)} w(H)$. Then $T$ is in $\mathcal{F N}$ by Lemma 2.1. Since $G^{(2 n)} \leq T$ it follows that $G / T$ is soluble.

There exist only finitely many $\mathbf{i} \in \mathbf{I}$ such that $w(\mathbf{i}) T / T \neq 1$. The theorem will be proved by induction on the number of such $n$-tuples $\mathbf{i}$.

Choose $\mathbf{i}=\left(i_{1}, \ldots, i_{n}\right) \in \mathbf{I}$ such that $w(\mathbf{i}) T / T \neq 1$ while $w(\mathbf{j}) T / T=1$ whenever $\mathbf{i}<\mathbf{j}$. It follows from Lemma 4.5 that $w(\mathbf{i}) T / T$ is abelian. Now we apply Lemma 4.4 and we obtain that $w(\mathbf{i})$ is in $\mathcal{F N}$. Let $N=w(\mathbf{i}) T$. Then induction on the number of $\mathbf{j} \in \mathbf{I}$ such that $w(\mathbf{j}) \not \leq N$ leads us to the conclusion that $w(G)$ is in $\mathcal{F N}$.

ACKNOWLEDGMENTS. This research was partially supported by Università di Padova (Progetto di Ricerca di Ateneo: "Invariable generation of groups"). The second author was also supported by GNSAGA (INDAM), and the third author by FAPDF and CNPq.

\section{References}

[1] C. Acciarri and P. Shumyatsky, Coverings of commutators in profinite groups, Rend. Sem. Mat. Univ. Padova, 137 (2017), 237-257.

[2] C. Acciarri and P. Shumyatsky, On profinite groups in which commutators are covered by finitely many subgroups, Math. Z. 274 (2013), 239-248.

[3] E. Detomi, M. Morigi and P. Shumyatsky, On countable coverings of word values in profinite groups, J. Pure Appl. Algebra 219 (2015), 1020-1030.

[4] E. Detomi, M. Morigi and P. Shumyatsky, On conciseness of words in profinite groups, J. Pure Appl. Algebra 220 (2016), 3010-3015.

[5] E. Detomi, M. Morigi and P. Shumyatsky, On groups covered by locally nilpotent subgroups, Ann. Mat. Pura Appl., 196 (2017), 1525-1535, doi: 10.1007/s10231-016-0627-y.

[6] D. Gorenstein, Finite Groups, Chelsea Publishing Company, New York, 1980.

[7] J. L. Kelley, General Topology, Van Nostrand, Toronto - New York - London, 1955.

[8] D. J .S. Robinson, A course in the theory of groups, Second edition, Graduate Texts in Mathematics, 80, Springer-Verlag, New York, 1996.

[9] A. Shalev, Combinatorial conditions in residually finite groups II, J. Algebra 157 (1993), 51-62.

[10] P. Shumyatsky, Verbal subgroups in residually finite groups, Q.J. Math. 51 (2000), 523-528.

[11] P. Shumyatsky, On profinite groups with commutators covered by nilpotent subgroups, Rev. Mat. Iberoam. 32 (2016), 1331-1339, doi: 10.4171/rmi/919.

[12] J.S.Wilson, Two-generator conditions for residually finite groups, Bull. London Math. Soc. 23 (1991), 239-248.

[13] J.S. Wilson and E. Zelmanov, Identities for Lie algebras of pro-p groups, J. Pure Appl. Algebra 81 (1992), 103-109.

[14] E. Zelmanov, Some problems in the theory of groups and Lie algebras (Russian), Mat. Sb. 180 (1989), 159-167; translation in Math. USSR-Sb. 66 (1990), 159-168. 
Dipartimento di Matematica, Università di Padova, Via Trieste 63, 35121 Padova, ITALY

Email address: detomi@math.unipd.it

Dipartimento di Matematica, Università di Bologna, Piazza di Porta San Donato 5. 40126 Bologna, ItAly

Email address: marta.morigi@unibo.it

Department of Mathematics, University of Brasilia, Brasilia-DF, 70910-900 Brazil

Email address: pavel@unb.br 